\title{
High Intensity Interval Training in Healthy Males Does not Improve Markers of Insulin Sensitivity
}

\author{
Douglas James Oberlin ${ }^{1}$, Jason Smith ${ }^{1}$, Kevin Ritsche ${ }^{1,2}$ and Laurie Wideman ${ }^{1}$ \\ 1. Department of Kinesiology, University of North Carolina Greensboro, Greensboro NC 27412, USA \\ 2. Department of Human Performance and Sport Science, Winston-Salem State University, Winston-Salem NC 27110, USA
}

\begin{abstract}
Purpose: Determine if three weeks of HIT (high-intensity interval training) improves fasting insulin sensitivity in healthy males. Methods: Participants were recreationally active ( $\geq 10$ hours per week) men between 18 and 35 years of age $(\mathrm{Ht}: 180 \pm 1.44$ cm; Wt: $85 \pm 2.95 \mathrm{~kg}$; BMI: $26.1 \pm 0.59 \mathrm{~kg} / \mathrm{m}^{2}$; body fat: $19.7 \pm 1.76 \%$ ). HIT training occurred 3 days weekly for 3 weeks, at intensities equivalent to $7.5 \%$ of body mass. Training volume increased weekly as follows: three sprints per session (week 1), four sprints per session (week 2), and five sprints per session (week 3). Fasting blood samples were collected at baseline and after each week and tested for glucose and insulin. A repeated measures ANOVA was used to measure changes in both fasting glucose and insulin concentrations as well as HOMA(IR) (homeostatic model assessment) and QUICKI (quantitative insulin sensitivity check index). Results: Values were in the normal range throughout the study and there were no significant improvements in glucose $(P=$ $0.346)$, insulin $(P=0.680)$, HOMA $(P=0.567)$, or QUICKI $(P=0.186)$, as a result of HIT. Conclusion: While HIT may be useful in maintaining insulin sensitivity in healthy males, 3-weeks of HIT did not further improve insulin sensitivity in this group.
\end{abstract}

Key words: Insulin resistance, exercise, glucose, QUICKI, HOMA, HIT, HIIT, SIT.

\section{Introduction}

The incidence of both obesity and T2D (type 2 diabetes) is rising in the United States [1, 2]. Both of these conditions increase the risk of mortality from cardiovascular disease as well as all-cause mortality [3, 4]. Therefore, it is important not only to develop new methods of treating individuals who are obese or diagnosed with $\mathrm{T} 2 \mathrm{D}$, but to prevent healthy individuals from developing these conditions. A potential avenue toward achieving this goal is through lifestyle management, including a healthy diet and regular physical activity or exercise.

Regular exercise has been shown to be effective at improving or maintaining insulin sensitivity, a metabolic risk factor associated with T2D [5-9]. The physical activity recommendation for adults is to achieve a minimum of 30 minutes of moderate intensity physical activity on five days each week or

Corresponding author: Douglas James Oberlin, M.S., research field: exercise physiology. E-mail: djoberli@uncg.edu. vigorous-intensity aerobic exercise for a minimum of 20 minutes on three days each week [10]. Unfortunately, only about 30 percent of adults in the United States are physically active based on these guidelines according to the National Health Interview Survey [11]. A commonly reported barrier to meeting the physical activity recommendations is a lack of time [12]. A viable option for increasing adherence to physical activity guidelines is to reduce the total time necessary to provide benefits. High intensity interval exercise provides a mechanism for reducing the total exercise time and may still promote positive metabolic outcomes related to insulin sensitivity.

Several studies have reported that short but high intensity exercise intervals are an effective strategy for improving insulin sensitivity [13-15]. A protocol using only 7.5 minutes of active exercise per week has been shown to improve time to exhaustion as well as citrate synthase content in muscles [16]. HIT (High intensity interval training) has even been shown to improve glycemic control (measured by 24 hour 
glucose levels) in individuals with T2D [14]. Although post-prandial (or post-challenge) glucose tolerance is improved by interval training, there does not seem to be a change in fasting indices of IS (insulin sensitivity) among healthy active individuals $[13,17]$. However, many interval training studies have only used a two week training period to examine these changes [13, 14, 17]. Perhaps healthy individuals require slightly longer training periods to further improve fasting markers of insulin sensitivity. Therefore, the current study attempts to extend the training protocol by an additional week, as well as increase the intensity of training each week. The primary purpose of the current study was to determine whether three weeks of a progressive HIT protocol would be sufficient to improve fasting markers of IS in healthy males.

\section{Materials and Methods}

\subsection{Subjects}

Participants in the current study were all healthy, recreationally active ( $\geq 10$ hours per week) Caucasian and African-American men between 18 and 35 years of age $(n=20)$. All subjects gave written informed consent prior to participation in this study, as approved by the Institutional Review Boards at the University of North Carolina at Greensboro, Winston-Salem State University and the University of Virginia. Subjects completed a detailed medical history form and a physical activity history form prior to participation. Exclusion criteria included: two or more cardiovascular disease risk factors, age greater than 35 years, less than 10 hours a week of physical activity, smokers, or a BMI $\geq 35$. Control subjects were obtained from another study with a matching target sample group $(n=9)$. Control subjects were matched by age, but not BMI, and were non-smokers, and were physically active 7 hours or less per week. Control subjects did not complete any exercise testing and agreed to make no changes to their lifestyle, but reported to the lab weekly to give fasting blood samples to be used as comparisons for the exercised group.

Height was recorded using a stadiometer and weight was recorded using an electronically calibrated scale (Seca, Vogel and Halke; Hamburg, Germany or Detecto Physician Scale; Webb City, Missouri).

\subsection{Wingate Testing}

Subjects completed a brief familiarization session on the cycle ergometer (Lode Excalibur Sport; Lode BV, Groningen, The Netherlands), for both acclimation as well as to adjust the bike for body size. Subjects returned to the lab within 120 to 168 hours following their familiarization session having fasted for 8-12 hours and refraining from exercise for at least 24 hours. Resting fasted blood samples were collected while the subject relaxed in a chair. After the blood draw was taken, the subjects moved to the cycle ergometer. The subjects first warmed up on the cycle ergometer, pedaling with no resistance for $5 \mathrm{~min}$. The subjects were then instructed to pedal at maximal capacity as the equivalent of $7.5 \%$ of their body mass was applied as resistance. The subjects pedaled against this resistance for $30 \mathrm{~s}$ after which the test ended and the subject was instructed to cool down. This procedure was repeated every Sunday during the training period to take blood samples and measure changes in power output values over time. This measure was used to assess any training adaptations, due to the use of Wingates in the HIT protocol.

\subsection{Training}

Participants underwent HIT on cycle ergometers using a protocol modified from Burgomaster et al. [16, 18]. Subjects would report to the lab 3 days each week (Monday, Wednesday, and Friday) for 3 weeks to complete training sessions within 36-48 hours of the initial wingate test. The intensity of the HIT was also equivalent to $7.5 \%$ of each subject's body mass, and each sprint lasted 30 seconds. The volume of training increased each week as follows: three sprints per 
session the first week, four sprints per session the second week, and five sprints per session the third week. Between sprints, the subjects continued to cycle at 50 watts for 4 minutes to allow for active recovery. Table 1 shows the time course for the training intervention.

\subsection{Blood Samples}

Blood samples were allowed to clot for 30 minutes at room temperature after being drawn. The samples were centrifuged at $3000 \mathrm{rpm}$ for 15 minutes at $4^{\circ} \mathrm{C}$ and serum was aliquoted into $1.5 \mathrm{~mL}$ microtubes and stored at $-80^{\circ} \mathrm{C}$ until completion of the study. Prior to assays, the serum samples were allowed to come to room temperature for approximately 1 hour. Mercodia Insulin ELISA (Mercodia; Uppsala, Sweden) was used for measurement of insulin and Cayman Glucose Colorimetric Assay Kit (Cayman Chemicals; Ann Arbor, Michigan) was used for measurement of glucose.

All samples were run in duplicate and the average value of the samples was used in analyses, and only accepted with a coefficient of variance less than $15 \%$ between samples. The inter assay CV (coefficient of variance) for insulin was $8.34 \%$ and for glucose was $2.56 \%$. The intra assay CV for insulin was $7.62 \%$ and for glucose was $4.37 \%$. HOMA(IR) and QUICKI were calculated and compared for each week of training and post training as they are commonly used as markers of fasting IS [19-21].

\subsection{Statistics}

PASW 18 (Armonk, NY) statistical software was used to run a two way repeated measures ANOVA to measure changes in plasma concentrations of glucose and insulin, as well as HOMA(IR) and QUICKI, at each week of training, and post training between groups. A one-way ANOVA was used to compare BMI between the control and exercise group. BMI was used as a covariate for the two-way repeated measures analysis. An alpha of 0.05 was set as the level of significance.

\section{Results}

There was no difference in age between the control and exercise group $(P=0.440, \mathrm{~F}=0.615)$, however, there was a significant difference in BMI $(P=0.005$, $\mathrm{F}=$ 9.297).

All exercise subjects $(N=20$; Age $=24.8 \pm 0.87$ years; $\mathrm{Ht}=180.0 \pm 1.44 \mathrm{~cm}$; Mass $=85.0 \pm 2.95 \mathrm{~kg}$ ) completed each of the HIT bouts and sample collections for each week of the study's progression. There was no change in BMI or aerobic capacity between the beginning and end of the study ( $P=0.6$ and $P=0.393$, respectively). There was no significant change in mean power output during the Wingate tests over the course of the study $(P=0.297, \mathrm{~F}=1.254)$. However, there was a significant improvement in peak power output from baseline $(P=0.001, \mathrm{~F}=8.907)$ (Fig. 1).

Because the data did not meet Mauchly's Test of Sphericity, a Greenhouse-Geisser correction was used for the analysis of the peak power output. A post-hoc test of main effects with a Bonferroni correction showed significance between the baseline measure of peak power output and each of the three subsequent

Table 1 Study Progression.

\begin{tabular}{|c|c|c|c|c|c|c|}
\hline Sunday & Monday & Tuesday & Wednesday & Thursday & Friday & Saturday \\
\hline Blood sample and Wingate test & $\begin{array}{l}4 \text { Wingates with } 4 \\
\text { minute recoveries }\end{array}$ & & $\begin{array}{l}4 \text { Wingates with } 4 \\
\text { minute recoveries }\end{array}$ & & $\begin{array}{l}4 \text { Wingates with } 4 \\
\text { minute recoveries }\end{array}$ & \\
\hline Blood sample and Wingate test & $\begin{array}{l}5 \text { Wingates with } 4 \\
\text { minute recoveries }\end{array}$ & & $\begin{array}{l}5 \text { Wingates with } 4 \\
\text { minute recoveries }\end{array}$ & & $\begin{array}{l}5 \text { Wingates with } 4 \\
\text { minute recoveries }\end{array}$ & \\
\hline Blood sample and Wingate test & $\begin{array}{l}6 \text { Wingates with } 4 \\
\text { minute recoveries }\end{array}$ & & $\begin{array}{l}6 \text { Wingates with } 4 \\
\text { minute recoveries }\end{array}$ & & $\begin{array}{l}6 \text { Wingates with } 4 \\
\text { minute recoveries }\end{array}$ & \\
\hline
\end{tabular}

It shows the number of sprints each week of training. All exercised subjects strictly followed this time table, control subjects only provided fasting blood samples. 


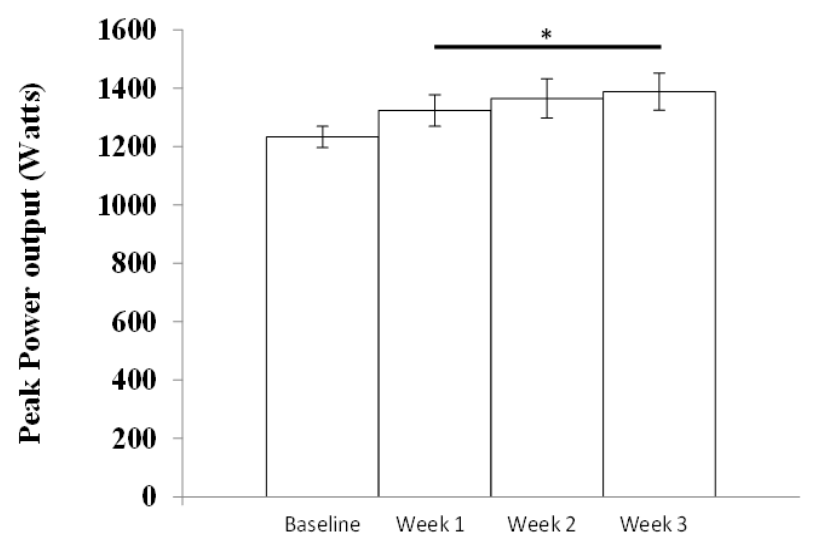

Fig. 1 Changes in Peak Power. It Illustrates changes in Peak Power Output on each week's Wingate Test. While there was a trend towards increased peak power output, there was only a significant difference between baseline (pre-training) and subsequent weeks. * indicates significant difference $(P<0.05)$ from baseline.

weeks ( $P=0.009,0.031$ and 0.005 , respectively).

Metabolic markers were measured from the fasting blood samples taken prior to each Wingate test or at weekly intervals for the control subjects (Table 1). There was no significant change in fasting glucose concentrations over the 3 weeks within either group $(P$ $=0.346, \mathrm{~F}=1.121)$, and there was no significant difference between groups $(P=0.058, \mathrm{~F}=3.934)$, however this approached significance (Fig. 2A). There was no significant change in fasting insulin concentrations over the 3 weeks within either group ( $P$ $=0.680, \mathrm{~F}=0.505)$, however, there was a significant difference between groups $(P=0.002, \mathrm{~F}=11.431)$ (Fig. 2B). Independent $t$ tests between groups for each time point revealed that the differences occurred at baseline, as well as time points 2 and $3(P=0.013, t=$
2.666; $P=0.029, t=2.556$ and $P=0.000, t=4.532$, respectively). There was also no significant change in HOMA or QUICKI over the 3 weeks within either group $(P=0.567, \mathrm{~F}=0.679$ and $P=0.186, \mathrm{~F}=1.644$, respectively). There were, however, differences between the two groups for both HOMA and QUICKI over the 3 weeks $(P=0.011, \mathrm{~F}=7.489$ and $P=0.00$, $\mathrm{F}=23.193$, respectively) (Fig. $2 \mathrm{C}$ and $\mathrm{D})$. Independent $t$ tests between groups for each time point showed that the differences occurred at only time point 3 and 4 for HOMA $(P=0.049, t=2.269$ and $P$ $=0.001 t=3.908$, respectively). However, QUICKI showed significant differences at each time point $(P=$ $0.21, \mathrm{t}=-2.455 ; P=0.16, t=-2.567 ; P=0.001, t=$ -3.761 and $P=0.001, t=-3.571)$.

\section{Discussion}

The primary goal of the current study was to determine whether 3 weeks of HIT would elicit improvements in fasting markers of IS rather than using the typical 2 weeks of HIT training. No statistically significant changes in markers of IS were observed during the fasting condition over the course of the study. There was, however, an improvement in peak power output showing some adaptation to the HIT training protocol. This study is unique in that it used greater than 2 weeks of HIT to examine training effects. Although there was not a significant change in fasting IS, there seemed to be no negative consequences to the training either.

Table 2 Group Differences. The mean values \pm SEM for blood and exercise markers for the HIT group before and after the 3 week HIT intervention.

\begin{tabular}{lllrr}
\hline \multirow{2}{*}{ Measure } & \multicolumn{3}{c}{ Value (mean \pm standard error) } \\
\cline { 2 - 5 } & \multicolumn{3}{c}{ Exercised Group } & \multicolumn{2}{c}{ Control Group } \\
\cline { 2 - 5 } & Pre-training & Post-training & Pre-study & Post-study \\
\hline Glucose $(\mathrm{mmol} / \mathrm{L})$ & $4.25 \pm 0.15$ & $4.40 \pm 0.12$ & $4.85 \pm 0.36$ & $4.28 \pm 0.34$ \\
Insulin $(\mathrm{mU} / \mathrm{I})$ & $6.71 \pm 0.56$ & $6.28 \pm 0.50$ & $15.86 \pm 4.52$ & $16.07 \pm 2.40$ \\
$\mathrm{BMI}\left(\mathrm{kg} / \mathrm{m}^{2}\right)$ & $26.1 \pm 0.59$ & $26.1 \pm 0.59$ & $23.18 \pm 0.65$ & - \\
$\mathrm{VO}_{2} \mathrm{max}(\mathrm{mL} / \mathrm{kg} / \mathrm{min})$ & $42.0 \pm 1.78$ & $42.6 \pm 1.92$ & - & - \\
Mean power (Watts) & $649.8 \pm 42.10$ & $606.6 \pm 31.47$ & - & - \\
Peak Power (Watts) & $1232.9 \pm 36.66$ & $1388.7 \pm 63.13 *$ & - & - \\
\hline
\end{tabular}

* indicates significant difference from baseline. 

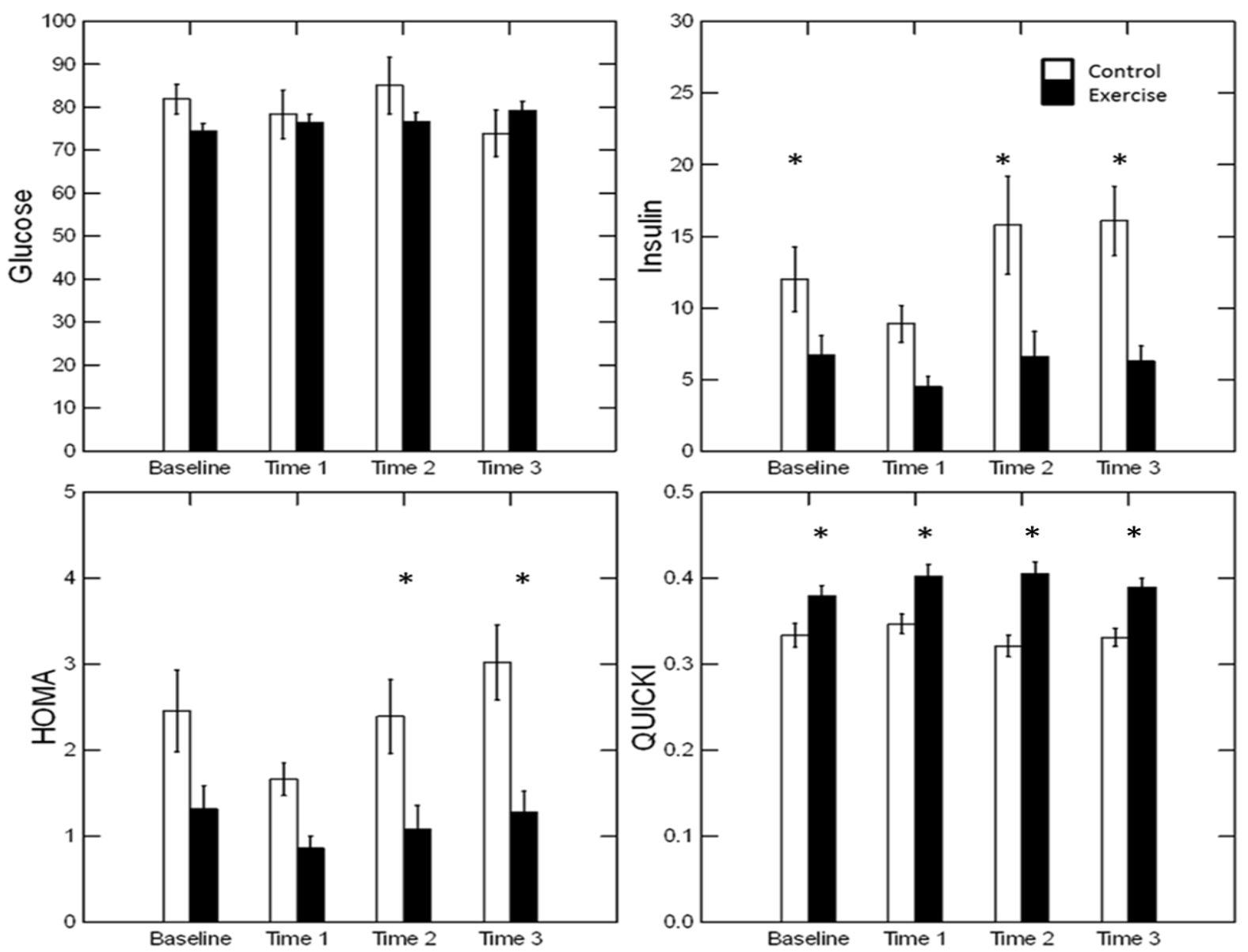

Fig. 3 Changes in Fasting Glucose and Insulin. It Shows the changes in fasting glucose in mg/dl. (A) fasting insulin in $\mathrm{mU} / \mathrm{I}$; (B) HOMA ( = (glucose (mmol/l)*insulin)/22.5); (C) QUICKI ( = 1/(log insulin + log glucose (mg/dl)); (D) between the HIT group and the control group at baseline, and over the 3 week intervention. * indicates significant difference between groups.

Neither the exercised subjects, nor the control subjects had significant changes in markers of fasting IS over the course of the study. However, the exercised group had significantly higher QUICKI values compared to the control group. Because the glucose concentrations in both groups were similar, these differences were most likely due to better insulin sensitivity in the exercised group. This can be observed in the lower fasting insulin concentrations amongst the exercised group when compared to the control group. Neither group showed any improvement or decrement over the course of the study, making it difficult to say whether the HIT was effective at maintaining these differences. A longer duration study may be required to determine whether or not HIT, compared to recreational activity, prevents loss of IS.
The current study examined fasting concentrations of insulin and glucose. The lack of changes in these measures may reflect a greater contribution of exercise to improved post-prandial glucose uptake, or an upper limit to the extent of improvement which can be induced in an already healthy population. Whether stimulated by contraction or insulin, skeletal muscles are capable of taking up glucose from the blood. However, in the fasting condition, regulation of blood glucose is more heavily influenced by hepatic glucose output in response to changes in the ratio of insulin and glucagon concentrations. While some studies have shown exercise to have an effect on hepatic glucose output, these effects may not be evident in individuals with already healthy fasting glucose and insulin concentrations [8, 13]. Additionally, the difference in the baseline fasting values in the current study suggest 
that even subtle difference in the volume of recreational activity (criteria for inclusion in control was $<7$ hours/week versus $<10$ hours/week for the exercise) is sufficient to induce significant differences in IS. What remains unclear is whether or not there is a lower level threshold for physical activity, below which benefits are lost in most individuals $(\mathrm{BMI} \leq 30)$, who do not become completely sedentary [19].

The method of estimation of IS may be a limiting factor in the lack of significant findings in the current study. Due to the effects of exercise on the skeletal muscle tissue and non-oxidative glucose disposal, changes may only be measurable using continuous glucose monitoring, oral glucose tolerance tests, intravenous glucose tolerance tests, frequently sampled glucose tolerance tests, or a hyper-insulinemic euglycemic clamp; where post-challenge glucose disposal is assessed. However, measuring the fasting concentrations of circulating glucose and insulin and calculating HOMA or QUICKI gives a more accurate measure of hepatic glucose regulation $[19,20]$. Also, because the fasting samples were taken approximately 48 hours after the last exercise session, the acute response of improved IS may have declined to the point where it could no longer be detected. Acute changes in insulin sensitivity have been reported to persist approximately 48 hours, but not beyond [22]. Therefore, any acute effects from the last training bout were not likely captured. More sensitive measurement techniques may be needed to detect any changes in a young, healthy, active population.

In addition to the measurement methods, it is possible that within a healthy active population, no changes are seen with increased exercise. Because all of the subjects in the current study began with healthy fasting levels of both insulin and glucose, it is unlikely that increasing exercise could suppress these concentrations further. The exercise group's QUICKI values were similar to those reported for elite athletes with high insulin sensitivity [23]. Indeed, lower concentrations of glucose in the fasting state may even be considered a negative adaptation because it could lead to fasting hypoglycemia. Because this population was already relatively fit, there was no change in peak $\mathrm{VO}_{2}$, however there was a significant improvement in peak power output over the 3 weeks due to the HIT [24]. Because this improvement occurred only after the first week, it was more likely an improved ability to perform the Wingate (learning effect), rather than a true training effect. This seems likely due to the lack of significant improvement in mean power output over the course of the rest of the study. These findings are in contrast to other studies which have used a similar protocol in individuals with T2D and seen robust changes in IS [14]. Even non-T2D obese older adults have shown a response to this intervention method, however the improvement was only detected 24 hours post exercise [15]. This is likely due to the low initial fitness level of individuals with T2D, allowing for a greater degree of improvement from baseline. If this is the case, differences may have been observed with this training protocol had a group of impaired glucose tolerant or impaired fasting glucose individuals been tested as well.

\section{Conclusion}

The current study has shown that 3 consecutive weeks of HIT was not adequate to further improve markers of fasting IS in an already healthy population. However, this may be taken as a positive result showing that HIT is a safe training modality which does not lead to fasting hypoglycemia. This may indicate that HIT is a potential exercise strategy to maintain IS within an already healthy population.

\section{Acknowledgments}

Dr. Paul Mellick made substantial contributions to the data collection during the current study.

\section{References}

[1] Wang, Y., Beydoun, M. A., Liang, L., Caballero, B. and Kumanyika, S. K. 2008. "Will All Americans Become 
Overweight or Obese? Estimating the Progression and Cost of the US Obesity Epidemic.” Obesity (Silver Spring) 16 (10): 2323-30.

[2] Yanovski, S. Z., and Yanovski, J. A. 2011. "Obesity Prevalence in the United States--up, Down, or Sideways?" N. Engl. J. Med. 364: 987-9.

[3] Saydah, S. H., Miret, M., Sung, J., Varas, C., Gause, D., and Brancati, F. L. 2001. "Postchallenge Hyperglycemia and Mortality in a National Sample of U.S. Adults." Diabetes Care 24 (8): 1397-402.

[4] Hajer, G. R., van Haeften, T. W. and Visseren, F. L. 2008. "Adipose Tissue Dysfunction in Obesity, Diabetes, and Vascular Diseases.” Eur. Heart J. 29 (24): 2959-71.

[5] Hawley, J. A., and Lessard, S. J. 2008. "Exercise Training-Induced Improvements in Insulin Action." Acta Physiol (Oxf). 192 (1): 127-35.

[6] Mikus, C. R., Oberlin, D. J., Libla, J. L., Taylor, A. M., Booth, F. W., and Thyfault, J. P. 2012. "Lowering Physical Activity Impairs Glycemic Control in Healthy Volunteers.” Med. Sci. Sports Exerc. 44 (2): 225-31.

[7] Mikus, C. R., Oberlin, D. J., Libla, J., Boyle, L. J., and Thyfault, J. P. 2012. "Glycaemic Control Is Improved by 7 Days of Aerobic Exercise Training in Patients with Type 2 Diabetes." Diabetologia 55 (5): 1417-23.

[8] Kirwan, J. P., Solomon, T. P., Wojta, D. M., Staten, M. A., and Holloszy, J. O. 2009. "Effects of 7 Days of Exercise Training on Insulin Sensitivity and Responsiveness in Type 2 Diabetes Mellitus." Am. J. Physiol. Endocrinol Metab. 297 (1): E151-6.

[9] Malin, S. K., Gerber, R., Chipkin, S. R., and Braun, B. 2012. "Independent and Combined Effects of Exercise Training and Metformin on Insulin Sensitivity in Individuals with Prediabetes." Diabetes Care 35 (1): 131-6.

[10] Haskell, W. L., Lee, I. M., Pate, R. R., Powell, K. E., Blair, S. N., Franklin, B. A., Macera, C. A., Heath, G. W., Thompson, P. D., and Bauman, A. 2007. "Physical Activity and Public Health: Updated Recommendation for Adults from the American College of Sports Medicine and the American Heart Association." Circulation 116 (9): 1081-93.

[11] Carlson, S. A., Densmore, D., Fulton, J. E., Yore, M. M., and Kohl, H. W. 2009. "Differences in Physical Activity Prevalence and Trends from 3 U.S. Surveillance Systems: Nhis, Nhanes, and Brfss." J. Phys. Act. Health 6 (Suppl. 1): S18-27.

[12] Shaw, S. M., Bonen, A., and McCabe, J. F. 1991. "Do More Constraints Mean Less Leisure? Examining the Relationship between Constraints and Participation.” Journal of Leisure Research 23 (4): 286-300.

[13] Babraj, J. A., Vollaard, N. B., Keast, C., Guppy, F. M., Cottrell, G., and Timmons, J. A. 2009. "Extremely Short
Duration High Intensity Interval Training Substantially Improves Insulin Action in Young Healthy Males." BMC Endocr. Disord 9: 3.

[14] Little, J. P., Gillen, J. B., Percival, M. E., Safdar, A., Tarnopolsky, M. A., Punthakee, Z., Jung, M. E., and Gibala, M. J. 2011. "Low-Volume High-Intensity Interval Training Reduces Hyperglycemia and Increases Muscle Mitochondrial Capacity in Patients with Type 2 Diabetes." J. Appl. Physiol. 111 (6): 1554-60.

[15] Whyte, L. J., Gill, J. M., and Cathcart, A. J. 2010. "Effect of 2 Weeks of Sprint Interval Training on Health-Related Outcomes in Sedentary Overweight/Obese Men." Metabolism 59 (10): 1421-8.

[16] Burgomaster, K. A., Hughes, S. C., Heigenhauser, G. J., Bradwell, S. N., and Gibala, M. J. 2005. "Six Sessions of Sprint Interval Training Increases Muscle Oxidative Potential and Cycle Endurance Capacity in Humans." $J$. Appl. Physiol. 98 (6): 1985-90.

[17] Richards, J. C., Johnson, T. K., Kuzma, J. N., Lonac, M. C., Schweder, M. M., Voyles, W. F., and Bell, C. 2010. "Short-Term Sprint Interval Training Increases Insulin Sensitivity in Healthy Adults but Does Not Affect the Thermogenic Response to Beta-Adrenergic Stimulation." J. Physiol. 588 (Pt 15): 2961-72.

[18] Gibala, M. J., Little, J. P., van Essen, M., Wilkin, G. P., Burgomaster, K. A., Safdar, A., Raha, S., and Tarnopolsky, M. A. 2006. "Short-Term Sprint Interval Versus Traditional Endurance Training: Similar Initial Adaptations in Human Skeletal Muscle and Exercise Performance.” J. Physiol. 575 (Pt 3): 901-11.

[19] Abdul-Ghani, M. A., Jenkinson, C. P., Richardson, D. K., Tripathy, D., and DeFronzo, R. A. 2006. "Insulin Secretion and Action in Subjects with Impaired Fasting Glucose and Impaired Glucose Tolerance: Results from the Veterans Administration Genetic Epidemiology Study." Diabetes 55 (5): 1430-5.

[20] Abdul-Ghani, M. A., Matsuda, M., Balas, B., and DeFronzo, R. A. 2007. "Muscle and Liver Insulin Resistance Indexes Derived from the Oral Glucose Tolerance Test." Diabetes Care 30 (1): 89-94.

[21] Katz, A., Nambi, S. S., Mather, K., Baron, A. D., Follmann, D. A., Sullivan, G., and Quon, M. J. 2000. "Quantitative Insulin Sensitivity Check Index: A Simple, Accurate Method for Assessing Insulin Sensitivity in Humans." J. Clin. Endocrinol. Metab. 85 (7): 2402-10.

[22] Henriksen, E. J. 2002. "Invited Review: Effects of Acute Exercise and Exercise Training on Insulin Resistance." $J$. Appl. Physiol. 93 (2): 788-96.

[23] Perseghin, G., Burska, A., Lattuada, G., Alberti, G., Costantino, F., Ragogna, F., Oggionni, S., Scollo, A., Terruzzi, I. and Luzi, L. 2006. "Increased Serum Resistin in Elite Endurance Athletes with High Insulin 
Sensitivity." Diabetologia 49 (8): 1893-900.

[24] Ritsche, K., Jason, S., Mellick, P., and Wideman, L. 2014.

"Acute Exercise-Induced Growth Hormone Is Attenuated in Response to Short-Term, High-Intensity Exercise Training." Journal of Exercise Physiology Online 17 (6): 1. 